\title{
AMERICAN SPIDER GENERA THERIDULA AND PARATHERIDULA (ARANEAE: THERIDIIDAE) ${ }^{1}$
}

\author{
By Herbert W. LeVI \\ Museum of Comparative Zoology
}

Since my revision (1954) of the genus Theridula in North America, no additional species have been found. In the meanwhile, however, I have been able to examine the types of South American species (except that of Theridula polita, which appears to be lost). Additional records of Paratheridula perniciosa have been obtained since I described the genus (1957), but no additional species. That Paratheridula perniciosa is a pantropical species is suggested not only by its wide distribution in the Americas, but also by the absence of related species. Its small size, inconspicuous appearance, and the fact that its epigynum is always covered by a secretion (which has to be removed to examine it), suggest that it may have been overlooked in collections.

I would like to thank Dr. G. Owen Evans and his staff at the British Museum (Natural History) for making the Keyserling types available for study. A National Science Foundation grant (G-43I7) permitted initial examination of theridiid types in European museums. The further researches were supported in part by Public Health Service Research Grant AI-or944 from the National Institute of Allergy and Infectious Diseases.

Misplaced SPecies: Theridula polita Mello-Leitão, 1947, Papéis Avulsos, Dept. Zool., São Paulo, 8(II) : I27, fig. I, +. Female holotype from Santa Cruz, Est. Paraná, Brazil, apparently lost, is probably a Dipoena judging by coloration, shape, and proportions of the species. The black stripe on the legs and the illustration of the epigynum suggest that it is $D$. militaris Chickering. The holotype of Theridula polita is not in the Mello-Leitão collection in Rio de Janeiro nor in São Paulo. Some Mello-Leitão types are alleged to be in the Museum Paranaense, but according to Mr. P. de Biasi it is not there either. The illustration of Mello-Leitão has been copied (Fig. 6).

\section{Theridula Emerton}

Theridula Emerton, 1882, Trans. Connecticut Acad. Sci. 6:25. Type species Theridion sphaerula Hentz, 1850 [ $=T$. opulenta (Walckenaer)], however Emerton misidentified the specimens which he examined; they belong to $T$. emertoni.

\footnotetext{
${ }^{1}$ Manuscript recived by the editor May 11, 1966
} 


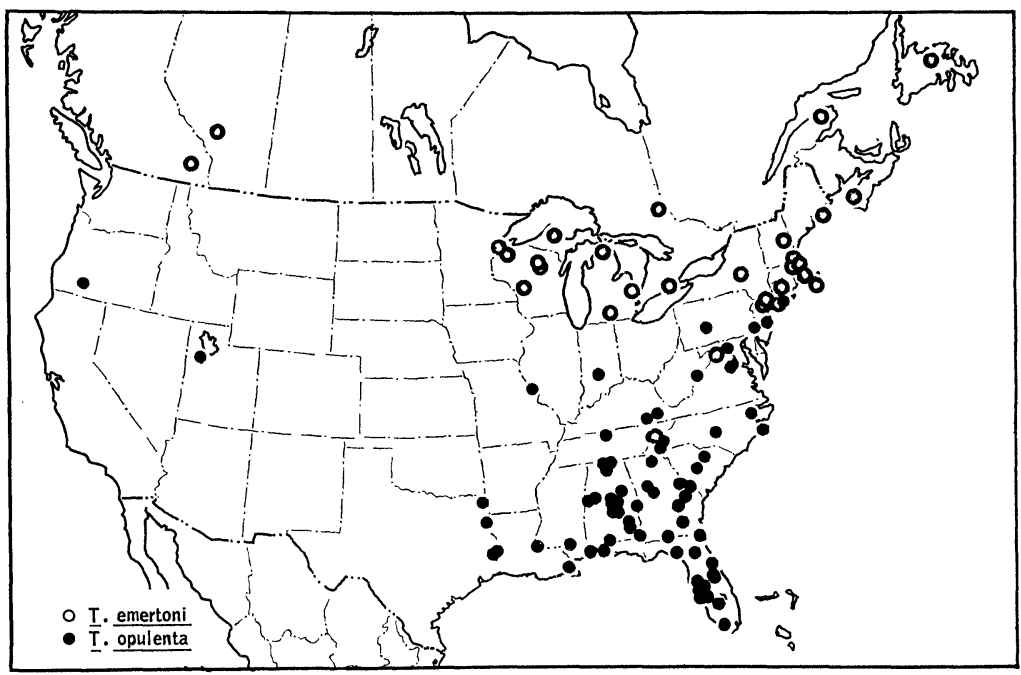

Map 1. Distribution of Theridula emertoni Levi and Theridula opulenta (Walckenaer).

Carapace as in Theridion, not modified. Chelicerae with two teeth on anterior margin, none posterior. First leg longest with patella and tibia $\mathrm{I} .2$ to $\mathrm{I} .5$ times carapace length. Abdomen wider than long in females, and lacking a colulus. Male palpus very simple, without conductor, median apophysis or radix. Haematodocha fastens both ends of tegulum to cymbium.

Key to Theridula species in the Americas

References to "figures" in lower case signify illustrations in Levi 1954, to "Figures" in upper case signify the ones in this paper.

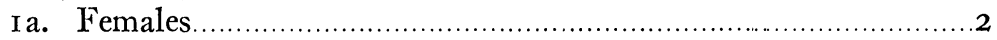

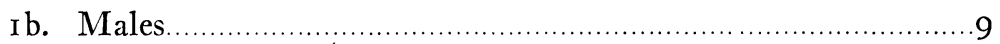

2a. Epigynum with two distinct circular openings (Figs. 2, 5) .......

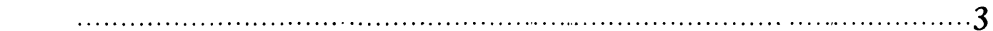

2b. Epigynum with humps or otherwise no distinct opening visible

3a. Connecting duct with one loop (fig. I9)

$3 \mathrm{~b}$. Connecting ducts not looped (Figs. I, 4)

gonygaster

4a. Openings connected by a posterior lip (Fig. 2)... multiguttata

4b. Openings on a hump not connected by a lip (Fig. 5). 


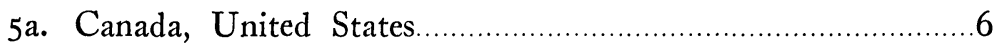

5b. Latin America ........................................................................

6a. Epigynum with a dark anterior semicircle (fig. 3).

emertoni

6b. Epigynum with a dark posterior transverse area (fig. II)

7a. Epigynum with two projecting bars (fig. 8).

opulenta

7b. Epigynum with humps

8a. Abdomen brown, usually with a black dorsal spot.......... faceta

8b. Abdomen black with white spots................................. puebla

9a. Palpal embolus as long as tegulum is high.............................. IO

9b. Palpal embolus shorter than tegulum high.......................... I I

IOa. Embolus tightly coiled (Fig. 3) …….................... nigerrima

Iob. Embolus loosely coiled (fig. I 8) ……………......... gonygaster

I Ia. Canada, United States ……………………....................... 2

I Ib. Latin America ...................................................................

12a. Palpal embolus straight with a notch on tip (fig. 9)

I2b. Palpal embolus undulating (fig. I) ............................. emertoni

opulenta

13a. Embolus as wide as long (fig. 23) ........................... puebla

I3b. Embolus longer than wide...............................................

I4a. Total length $\mathrm{I} .4$ to $1.9 \mathrm{~mm}$...................................... faceta

I4b. Total length about $2.5 \mathrm{~mm}$.......................................... casas

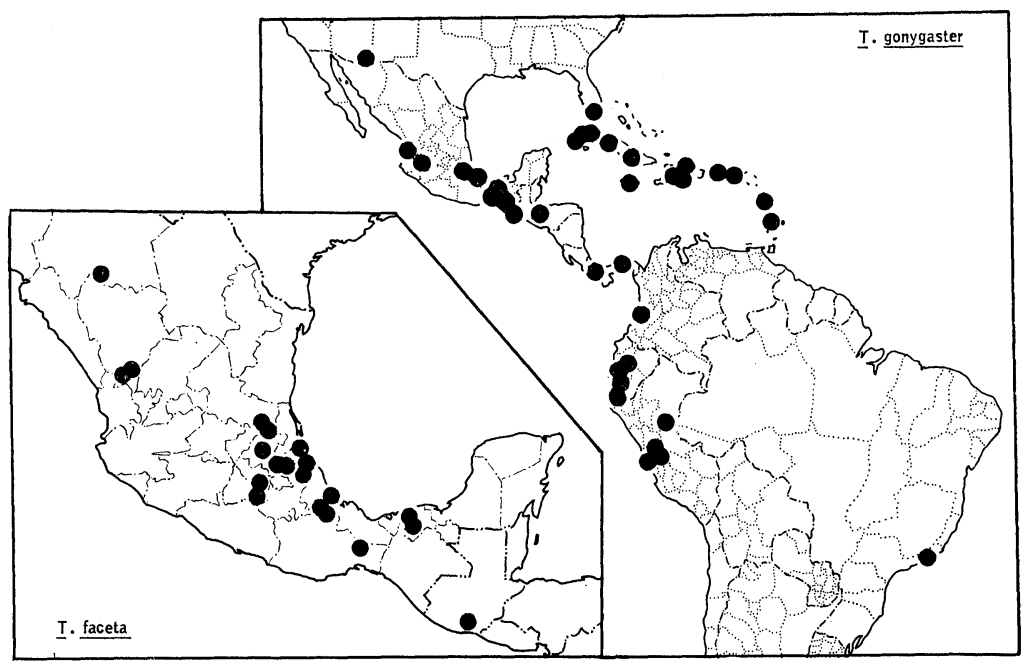

Map 2. Distribution of Theridula faceta (O. P.-Cambridge) and Theridula gonygaster (Simon). 


\section{Theridula emertoni Levi Map I}

Additional record. Newfoundland. Junction Pond, Notre Dame Camp, I3 July I96r, 우 (C. P. Alexander).

\section{Theridula opuienta (Walckenaer) Map I}

Additional record. United States. Oregon: Crater Lake Natl. Park, I5 June I947, 우 (B. Malkin and S. Sargent).

$$
\begin{gathered}
\text { Theridula faceta (O. P. - Cambridge) } \\
\text { Map } 2
\end{gathered}
$$

Additional record. Mexico. Tabasco: $3.2 \mathrm{~km} \mathrm{NE}$ of Comalcalo.

\section{Theridula puebla Levi \\ Map 3}

Additional records. Panama. Canal Zone: Madden Dam, 8 Aug. I939 (A. M. Chickering); Barro Colorado Island, several records (A. M. Chickering). Boquete, several records (A. M. Chickering).
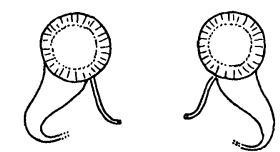
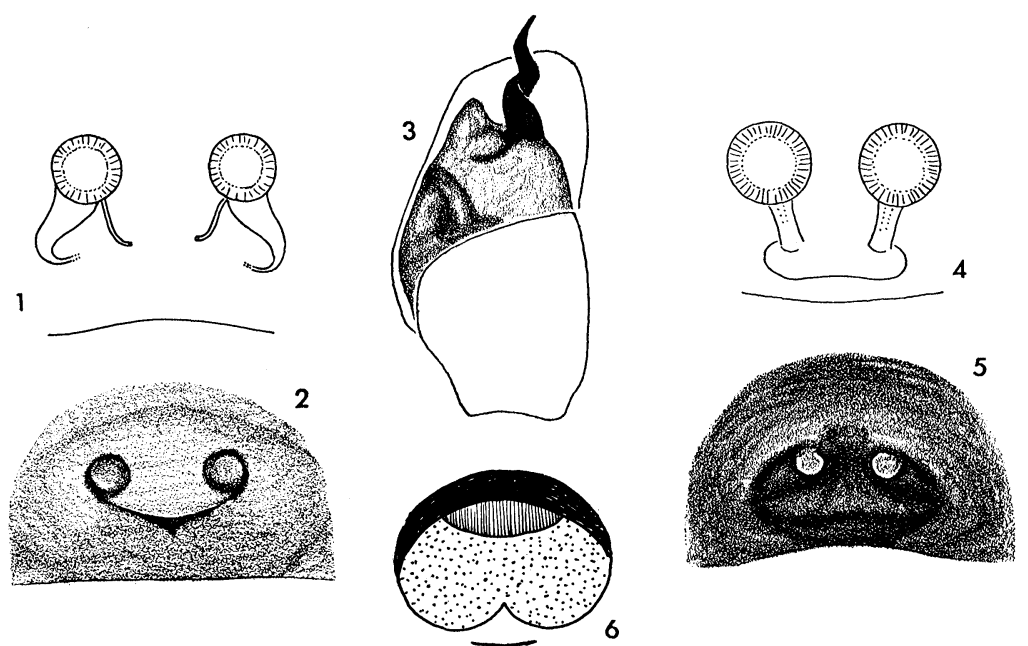

Figs. 1, 2. Theridula multiguttata Keyserling. 1. Female genitalia, dorsal view. 2. Epigynum.

Figs. 3-5. T. nigerrima (Petrunkevitch). 3. Left palpus. 4. Female genitalia, dorsal view. 5. Epigynum.

Fig. 6. T. polita Mello-Leitao, epigynum after Mello-Leitão. 


\section{Theridula gonygaster (Simon) Map 2}

Theridium gonygaster Simon, 1873, Mém. Soc. Roy. Sci. Liège, (2) 5:109. Male lectotype designated by Levi, 1954, in the Muséum National d'Histoire Naturelle, Paris.

Theridula gonygaster, - Levi, 1954, Trans. Amer. Microscop. Soc., 73 :340, figs. 18-22, 우 $\hat{o}$.

Distribution. Worldwide tropical. In America from Arizona, southern Florida, eastern Mexico, West Indies to Rio de Janeiro, Brazil.

Additional records. United States. Arizona: Cochise Co. Cave Creek Canyon. Columbia. Valle: Buenaventura, 4 Nov. I950 (E. I. Schlinger, E. S. Ross). Ecuador. Guayas: Milagro (H. E., D. L. Frizzell); Guayaquil (Landes, H. E., D. L. Frizzell). El Oro: $5 \mathrm{~km}$ above Pasaje (R. Walls). Peru. Piura: Sulana, banana grove (H. E., D. L. Frizzell) ; Higuerón, Las Lomas (H. E., D. L. Frizzell). Lima: San Antonio (E. I. Schlinger, E. S. Ross).

\section{Theridula nigerrima (Petrunkevitch) \\ Figures 3-5, Map 3}

Dipoena nigra Keyserling, 1886, Die Spinnen Amerikas, Theridiidae, 2(2): 43, pl. 12, fig. 158, of $\hat{o}$. Female lectotype here designated from Pumamarca [?Pumamanta, Junin], Peru, in the Polish Academy of Sciences, Warsaw; examined. Name preoccupied by D. nigra Emerton, 1882.

Dipoena nigerrima Petrunkevitch, 1911, Bull. Amer. Mus. Nat. Hist., 29: 174. New name for D. nigra Keyserling, preoccupied.

Diagnosis. The openings of the epigynum are on the anterior edge of a sclerotized plate (Fig. 5), quite different from $T$. gonygaster which lacks this plate. The connecting canals seem straight and short (Fig. 4). The shape of the narrow pointed embolus (Fig. 3) separates $T$. nigerrima from $T$. gonygaster.

Records. Ecuador. Azuay: Cuenca, 3 Apr. 1942, o (H. E., D. L. Frizzell). Peru. Cuzco: Machu Picchu, $3000 \mathrm{~m}$ elev., 6 Mar. I947, 2 o (J. C. Pallister).

\section{Theridula multiguttata Keyserling Figures I, 2. Map 3}

Theridula multiguttata Keyserling, 1886, Die Spinnen Amerikas, Theridiidae, 2: 258, pl. 21, fig. 306, ․ Female holotype from Blumenau, [Santa Catarina], Brazil, in the British Museum (Natural History); examined.

The shorter straight connecting ducts (Fig. 4) separate this species from $T$. gonygaster. 


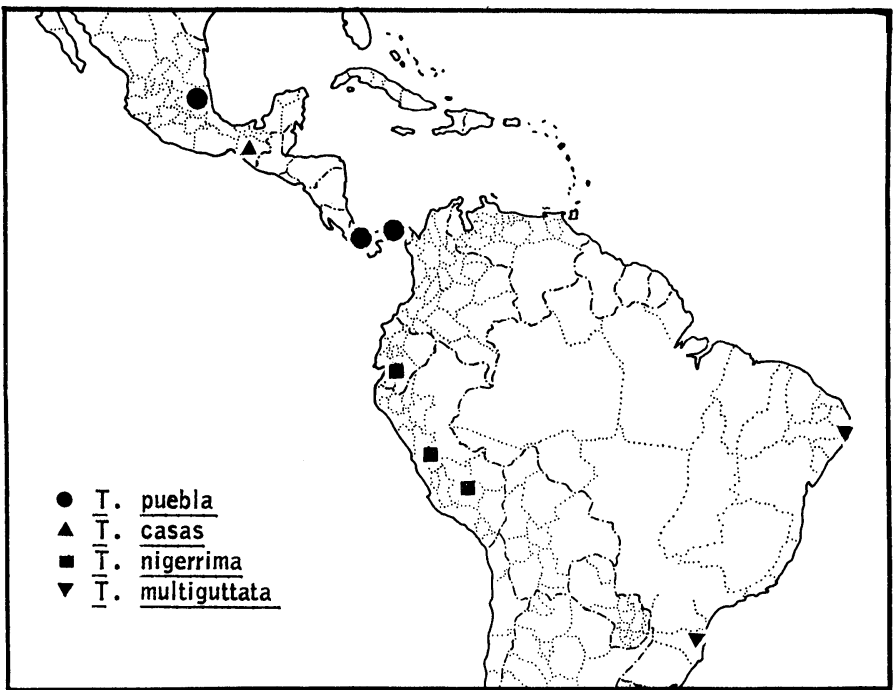

Map 3. Distribution of Theridula puebla Levi, T. casas Levi, T. nigerrima (Petrunkevitch), and $T$. multiguttata Keyserling.

Record. Brazil. Pernambuco: Recife (in the Senckenberg Museum).

\section{Paratheridula Levi}

Paratheridula Levi, 1957, Trans. Amer. Microscop. Soc., 76:105. Type species by original designation, Mysmena quadrimaculata Banks [=Paratheridula perniciosa (Keyserling)].

Carapace as in Theridion, not modified. Chelicerae with two teeth on anterior margin, a small tooth on posterior of female. First leg longest, patella and tibia I.3 to I.4 times carapace length. Abdomen subspherical and without colulus. Male palpus very simple without conductor, median apophysis or radix, and having only a basal hematodocha.

The single species known is widely distributed; it is suspected that it is introduced.

\section{Paratheridula perniciosa (Keyserling) Map 4}

Theridium perniciosum Keyserling, 1886, Die Spinnen Amerikas, Theridiidae, $2(2):$ 233, pl. 20, fig. 288, +. Female type from Blumenau, [Est. Santa Catarina], Brazil, in the British Museum (Natural History); examined. 


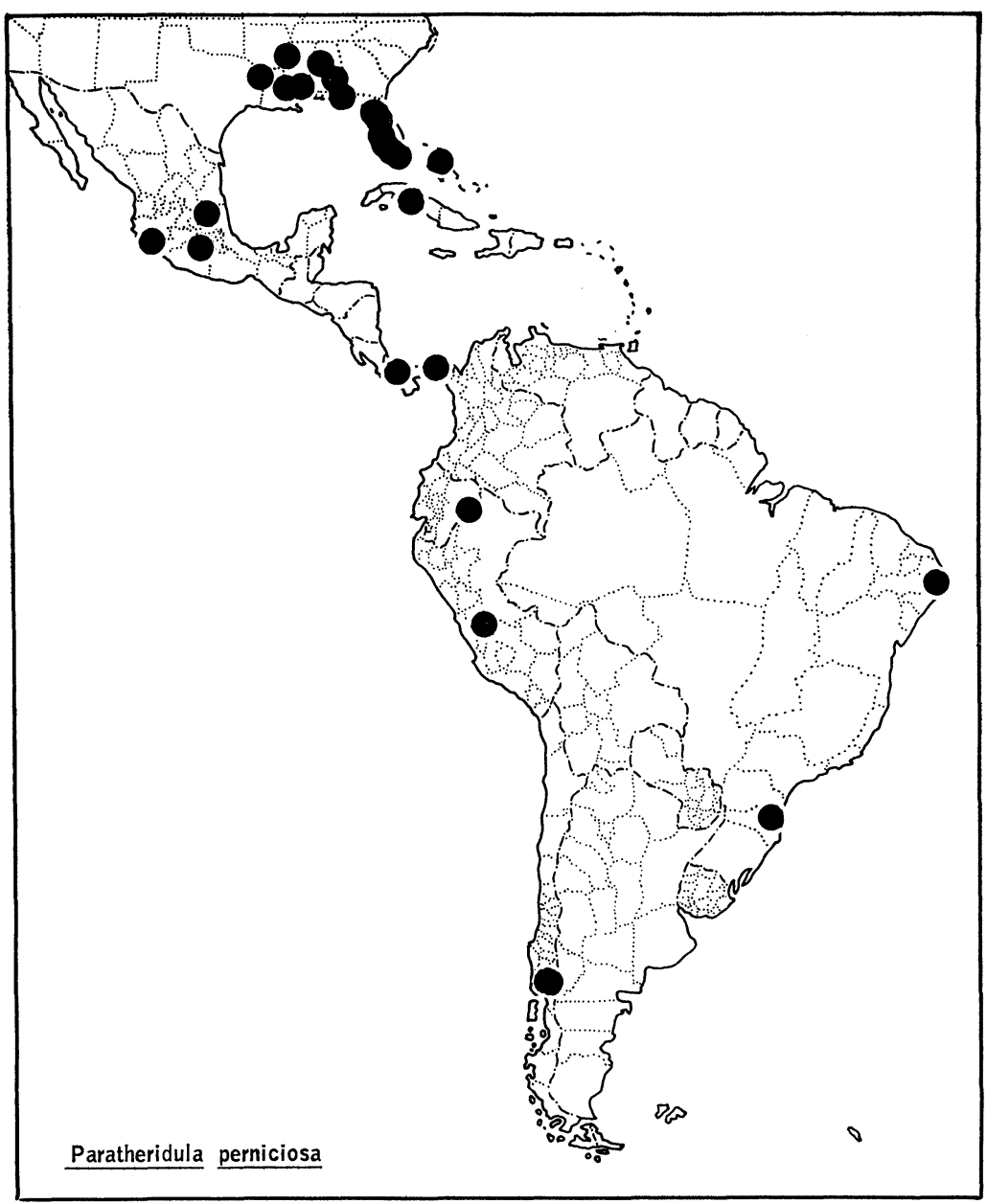

Map 4. Distribution of Paratheridula perniciosa (Keyserling). 
Mysmena quadrimaculata Banks, 1896, Trans. Amer. Entomol. Soc., 23: 66. Female holotype from Punta Gorda, Florida, in the Museum of Comparative Zoology.

Faratheridula quadrimaculata, - Levi, 1957, Trans. Amer. Microscop. Soc., 74: 106, figs. 1-6, map.

Distribution. Gulf states, Mexico, Central America, West Indies, South America to Chile, possibly pantropical.

Additional records. Mexico. San Luis Potosí: Valles, July 1956 (W. J. Gertsch, V. Roth). Cuba. Villas: Soledad. Bahama Island. New Providence (J. Greenway). Ecuador. Napo-Pastaza: Río Topo, June I943 (H. E., D. L. Frizzell). Peru. Junín: San Ramón, $800 \mathrm{~m}$ (H. W. Levi). Brazil. Pernambuco: Recife. Chile. Osorno: Valley Forest, $18 \mathrm{~km} \mathrm{~W}$ of Purranque, Jan. I95 I (E. S. Ross, A. E. Schlinger); ro km E of Puyehue, Jan. I95 I (E. S. Ross, A. E. Schlinger).

\section{References Cited}

LEVI, H. W.

1954. The spider genus Theridula in North and Central America and the West Indies. Trans. Amer. Microscop. Soc. 73: 331-343.

1957. The North American spider genera Paratheridula, Tekellina, Pholcomma, and Archerius. Ibid. 76: 105-115. 

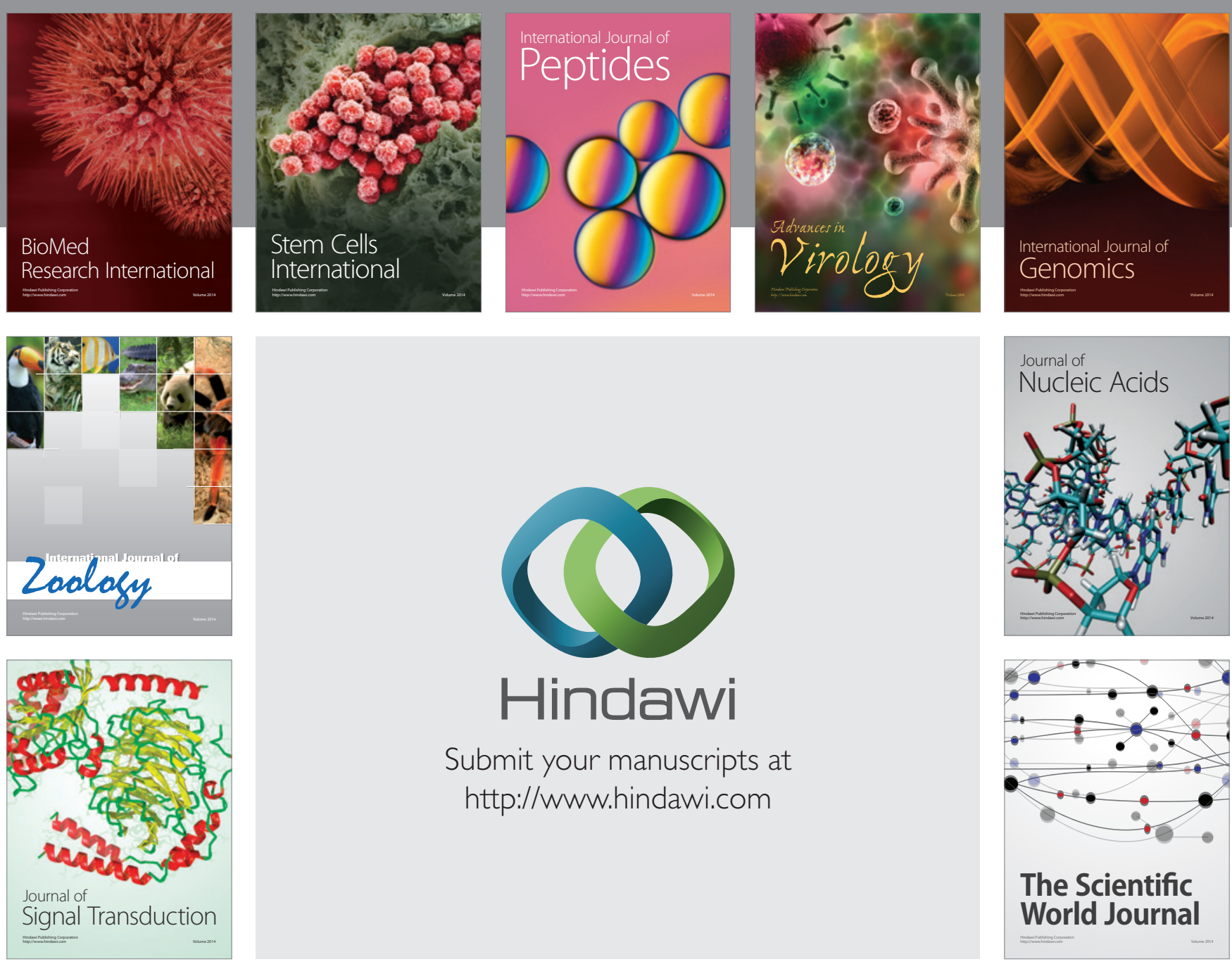

Submit your manuscripts at

http://www.hindawi.com
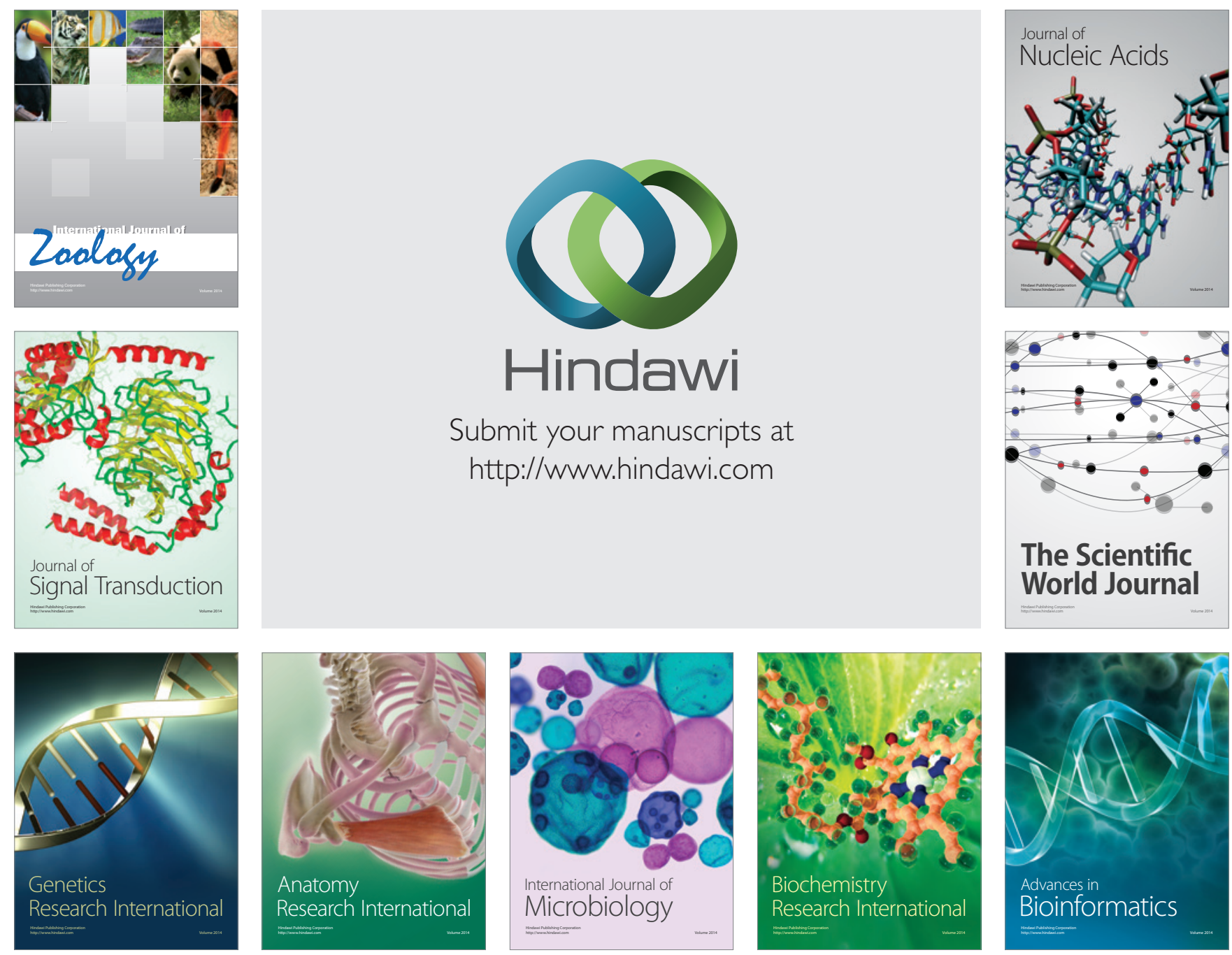

The Scientific World Journal
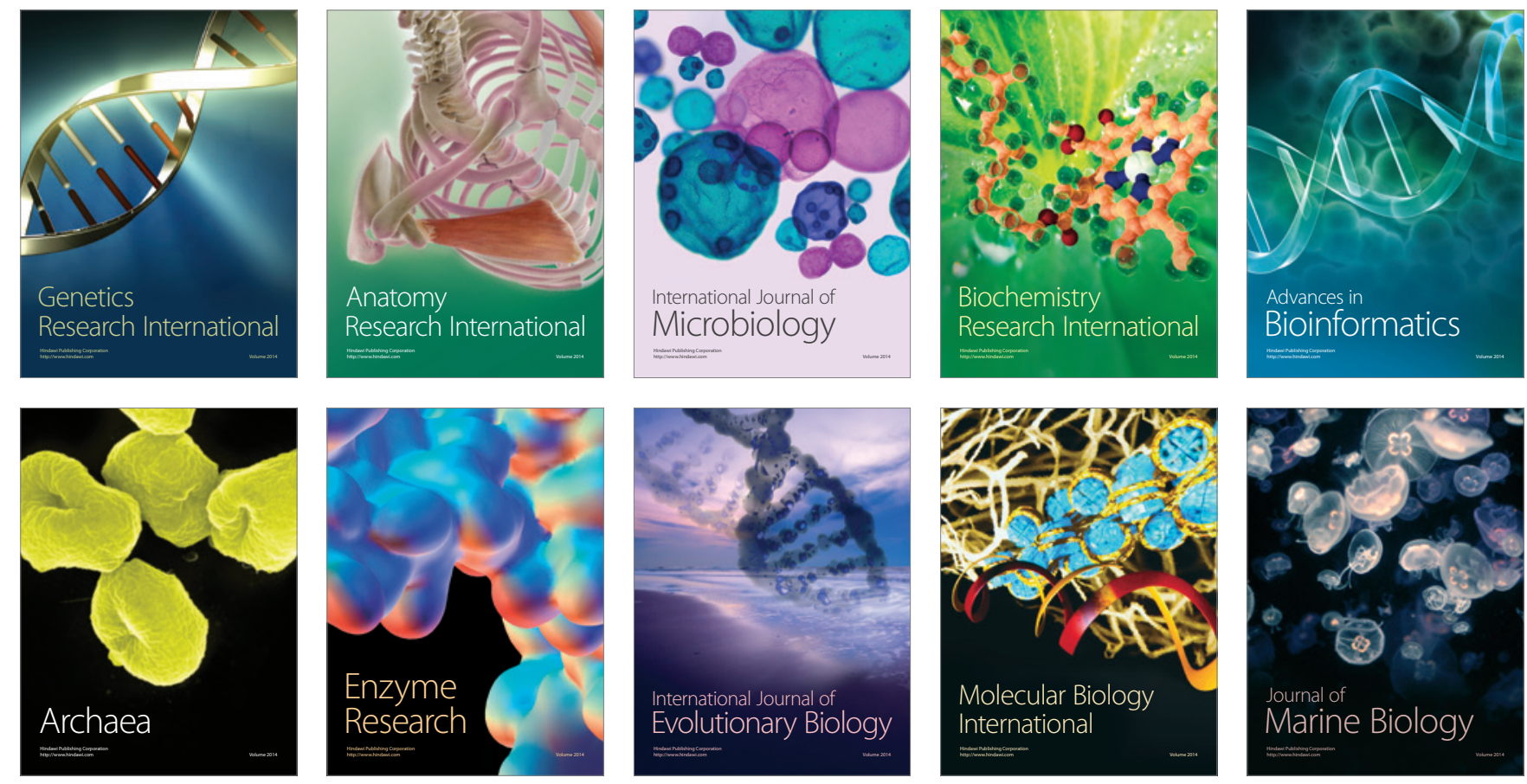